\title{
Low temperature thermomagnetic properties of very heavy fermions suitable for adiabatic demagnetization refrigeration
}

\author{
Julian G. Sereni \\ Physics Department, CAB-CNEA, CONICET, 8400 San Carlos de Bariloche, Argentina
}

(Dated: July 24, 2018)

\begin{abstract}
With the aim of improving the performance of classical paramagnetic salts for adiabatic refrigeration processes at the sub-Kelvin range, relevant thermodynamic parameters of some new $\mathrm{Yb}$ based intermetallic compounds are analyzed and compared. Two alternative potential applications are recognized, like those requiring fixed temperature reference points to be reached applying low intensity magnetic fields and those requiring controlled thermal drift for temperature dependent studies. Different thermomagnetic entropy $S(T, B)$ trajectories were identified depending on respective specific heat behaviors at very low temperature. To gain insight into the criteria to be used for a proper choice of suitable materials in respective applications, some simple relationships are proposed to facilitate a comparative description of their magnetocaloric behavior, including the referent Cerium-Magnesium-Nitride $(\mathrm{CMN})$ salt in these comparisons.
\end{abstract}

\section{INTRODUCTION}

Technical requirements imposed by photo-detectors installed in orbital satellites [1] and the shortage of $\mathrm{He}^{4} /$ $\mathrm{He}^{3}$ gases due to an increasing demand and reducing supply 2] have powered the search of new materials able to improve the cryo-performance of classical paramagnetic salts 3 for adiabatic demagnetization refrigeration (ADR) processes 4]. These novel demands require cryo-materials suitable to operate under extreme conditions at the sub-Kelvin range of temperature. Two typical demands can be mentioned, one of them requiring a thermally stable cryo-source tuned at a precise operating temperature, i.e. acting as fixed thermal point (FTP). The other corresponds to provide a controlled thermal drift (CTD), maximizing the heating absorption in an extended range of temperature.

Apart from these specific potential needs, some common demands arise like: to have 'friendly' synthesis, good chemical stability, high thermal conductivity, low eddy currents and large volumetric entropy. Moreover, to have good performance at low applied field together with mechanical hardiness and to support dehydration [4] under vacuum conditions are 'sine qua non' conditions for satellite applications.

As it can be seen in Fig. 17, the ADR thermodynamic cycle can be described by three successive steps. The first corresponds to the application of magnetic field in isothermic conditions with a heat transference $Q_{t h}$ to the thermal bath at the initial temperature $T_{i}$. In this branch, depicted by a vertical arrow, the heat given out depends on the entropy reduction induced by increasing the applied field as: $Q_{t h}=\left.T_{i} \Delta S_{m}(B)\right|_{T_{i}}$.

The following step consists in the adiabatic cooling process where the cooling window $\Delta T=T_{i}-T_{f}$ (with $T_{f}=$ the final temperatures) is also included in Fig. 1 a as an horizontal arrow where the thermodynamic equation: $d S(T, B)=\left.(\partial S / \partial T)\right|_{B} d T+\left.(\partial S / \partial B)\right|_{T} d B=0$ ap- plies. This formula can be written including measurable parameters as $d S(T, B)=C_{m} / T d T+\partial M / \partial T d B=0$ because $\partial S / \partial T=C_{m} / T$ and $\partial S /\left.\partial B\right|_{T}=\partial M /\left.\partial T\right|_{B}$.

Along the third step the cryo-material is devoted to stabilize or control the temperature drift by absorbing heat $Q_{a b s}$. This heat is represented in Fig. 1 1 a by the shadowed area: $Q_{a b s}=\int_{S_{i}}^{S_{f}} T d S_{m}$. Since this parameter depends on the heat capacity along the temperature range, it corresponds to the enthalpy variation at $B=0$ because $T \partial S_{m}=C_{m} \partial T$. From this expression it becomes clear that materials without magnetic order but with robust magnetic moments are relevant for ARD purposes. These two conditions, that look contradictory, are realized in some recently discovered materials.

Since $C_{m} / T$ is proportional to the density of excitations, the rich family of intermetallic heavy Fermions (HF) provides asignificant number of candidates for ADR applications. Among them are the recently synthesized Yb-intermetallic compounds [5 10, some of which have been tested as proper candidates for the proposed improvements because of their very large $C_{m} /\left.T\right|_{T \rightarrow 0}$ values. These compounds were labeled as very $\mathrm{HF}$ (VHF) 11 because they largely exceed the values of classical HF [12]. According to their physical behavior within the $\mathrm{mK}$ range three well defined types of $C_{m}(T) / T$ behaviors were recognized [13: (I) the actual VHF with $C_{m} /\left.T\right|_{T \rightarrow 0}>10 \mathrm{~J} / \mathrm{molK}^{2}$, (II) those showing a constant $C_{m} /\left.T\right|_{T \rightarrow 0}$ 'plateau' around $7 \mathrm{~J} / \mathrm{molK}^{2}$ below a characteristic temperature $T^{*}$ and (III) those belonging to the non-Fermi-liquid (NFL) family [12] because of their $C_{m}(T) / T \propto-\ln \left(T / T_{0}\right)$ dependence.

All these paramagnetic materials do not order magnetically down to about $200 \mathrm{mK}$ despite of their robust magnetic moments, providing large $\partial M / \partial T$ coefficients. As mentioned before, the entropy computed as: $S_{m}(T)=\int C_{m} / T d T$ is the basic parameter for the study of ARD processes because its thermal trajectory characterizes the efficiency and applicability of 


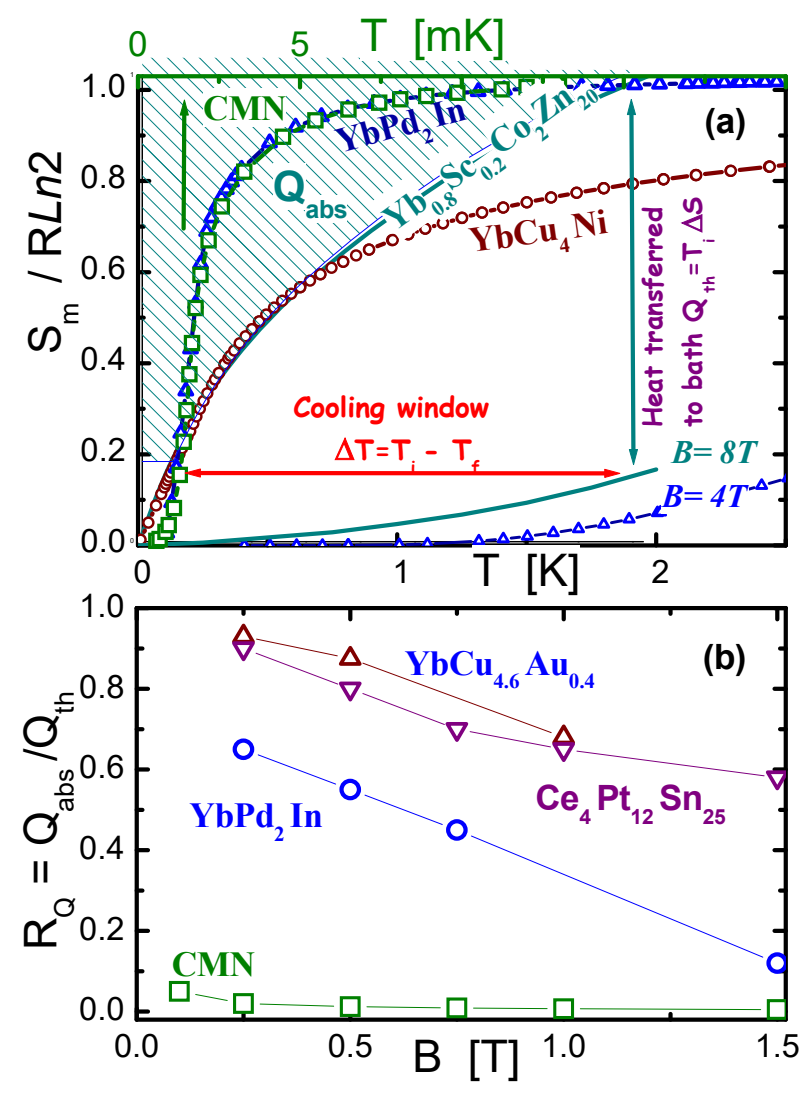

FIG. 1. (a) Exemplary $S_{m}(T)$ trajectories for three different types of behaviors [6] 8 . CMN salt (upper $T$ scale) is included for comparison 3. Vertical arrow: heat transferred to the bath $Q_{t h}$. Horizontal arrow: cooling window $\Delta T$. Shadowed triangle (top left) represents the heat absorbed $Q_{a b s}$ by these materials in the process of thermal stabilization taking as reference the $(\mathrm{Yb}, \mathrm{Sc}) \mathrm{Co}_{2} \mathrm{Zn}_{20}$ system [7]. (b) Heats ratio $R_{Q}=Q_{a b s} / Q_{t h}$ as a function of magnetic field evaluated from $T_{i}=1 \mathrm{~K}$.

each cryo-material at the 'sub-Kelvin' range.

The scope of this work focuses mainly on the analysis and comparison of the thermomagnetic properties of new intermetallic compounds exhibiting different behaviors which can be applied in alternative applications. In the search of a practical criteria to identify the mot appropriated material for each application, some simple relationships are proposed to facilitate a comparative description of their magnetocaloric behavior. The well known salt Cerium-Magnesium-Nitride (CMN), largely used for ADR processes because it remains paramagnetic down to $2 \mathrm{mK}[3,16$, is also included in this study as a reference to classical salts.

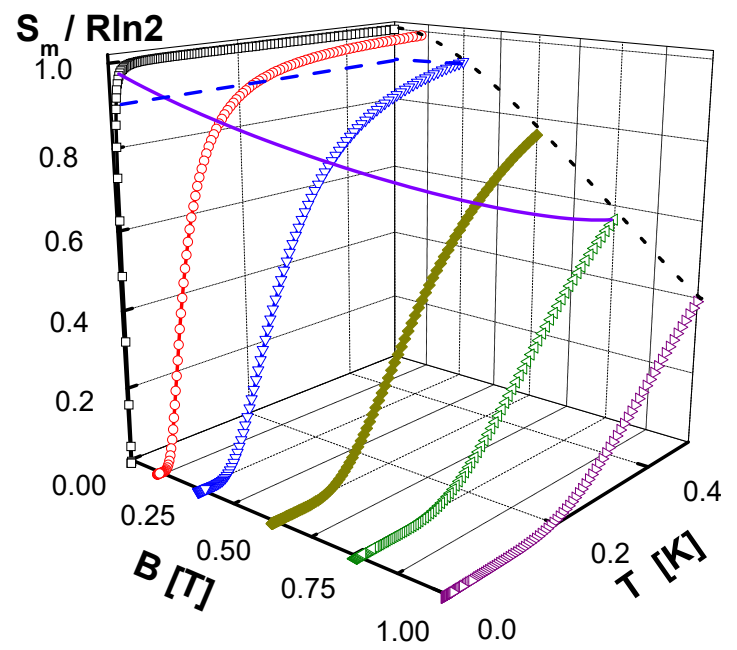

FIG. 2. Exemplary $S_{m}(T, B)$ trajectories at different fields for $\mathrm{CMN}$, after 3 in a $3 \mathrm{D}$ representation. Continuous curve indicates the maximum curvature of the $S_{m}(T, B)$ trajectory, dashed line the thermal window $\Delta T=T_{i}-T_{f}$ for $B=0.25 \mathrm{~T}$ and dotted curve the $\partial S_{m} /\left.\partial B\right|_{T_{i}}$ variation at $T_{i}=0.4 \mathrm{~K}$.

\section{ANALYSIS OF THERMOMAGNETIC PROPERTIES}

As expected, FTP and CTD applications require different types of $S_{m}(T)$ trajectories to optimize respective ADR processes. In Fig. 117, three exemplary types of trajectories are depicted showing that they can be sorted between those suitable as FTP providers and two alternatives of moderate increase of $S_{m}(T)$ more indicated for CTD operation conditions.

In the figure one may appreciate how the $S_{m}(T)$ trajectory of CMN matches with that of $\mathrm{YbPd}{ }_{2} \mathrm{In}$ by simply normalizing respective scales of temperature. Therefore this reference salt can be included in group (I) like a member of the $\mathrm{YbT}_{2} \mathrm{X}$ family (where $\mathrm{T}=\mathrm{Pd}$ and $\mathrm{Pt}$ and $\mathrm{X}=\mathrm{In}$ and $\mathrm{Sn}$ ). These compounds are better indicated for FTP processes because of their steep $\partial S / \partial T=C_{m} / T$ slope allows to tune them within a relative narrow thermal window around the required working range, e.g. according to satellite photon-detectors requirements. Differently, $\mathrm{YbCu}_{4} \mathrm{Ni}$ and $(\mathrm{Yb}, \mathrm{Sc}) \mathrm{Co}_{2} \mathrm{Zn}_{20}$, also included in Fig. 1 a belong to groups (II) and (III) respectively and better apply for CTD requirements because of their moderate slope allows a more convenient heat absorption more homogeneously distributed in temperature. Within group (II) one can mention $\mathrm{YbCu}_{4} \mathrm{Ni}[8]$ which represents the family of compounds showing a $C_{m} /\left.T\right|_{T \rightarrow 0} \approx$ $7.5 \pm 0.5 \mathrm{~J} / \mathrm{mol} \mathrm{K}^{2}$ 'plateau' followed by a common power 
law above a change of regime at $T=T^{*}$ [11. The compounds included in group (III) are characterized by showing NFL behavior, like $(\mathrm{Yb}, \mathrm{Sc}) \mathrm{Co}_{2} \mathrm{Zn}_{20}[7$ and $\mathrm{CeNi}_{9-x} \mathrm{~T}_{x}$ (where $\mathrm{T}=\mathrm{Co}[14$ with $x=0.1$, and $\mathrm{T}=$ $\mathrm{Cu}$ with $x=0.4$ [15] respectively).

One of the basic questions to be addressed in the ADR planning is the choice of the more appropriated material for each specific application. Since the number of involved parameters provides a rich spectrum of possibilities for an optimal application, the identification of physical quantities for a convenient comparison between different systems is advisable. Some proposition can be already extracted from Fig. 1a involving the mentioned heat transfers involved in the thermodynamic cycle: $Q_{t h}$ and $Q_{a b s}$. A sort a efficiency in the heat management can be defined by the capability of the system to absorb heat in confront to the amount previously transferred to the bathat different fields through the ratio $R_{Q}(B)=Q_{a b s}(B) / Q_{t h}(B)$. In Fig. 1b this comparison is presented for four representative compounds of different types of $S_{m}(T, B)$ trajectories whose data are available in the literature as a function of applied fields within the $B \leq 1.5 \mathrm{~T}$ range. For practical reasons this comparison was done taking $T_{i}=1 \mathrm{~K}$, with the magnetic field within an eventual satellite application range.

This comparison includes the $R_{Q}(B)$ ratio obtained from compounds belonging to groups (I): $\mathrm{CMN}, \mathrm{YbPd}_{2} \mathrm{In}$ and $\mathrm{Ce}_{4} \mathrm{Pt}_{12} \mathrm{Sn}_{25}$, and group (II): $\mathrm{YbCu}_{4.6} \mathrm{Au}_{0.4}$. There, one may appreciate that a better ratio is provided by a system with lower $\partial S / \partial T$ slope (like $\mathrm{YbCu}_{4.6} \mathrm{Au}_{0.4}$ ) than by one with a larger slope (like CMN). An intermediate alternative is provided by the VHF $\mathrm{YbPd}_{2} \mathrm{In}$. From this first comparison one learns that the compound reaching the largest $C_{m} / T$ value at very low temperature is not the best for the heat absorption in a CDT process. Nevertheless, their $Q_{a b s}$ values can be considerably increased by tuning $T_{f}$ at higher values by not reducing the field down to zero.

Before to proceed for further comparisons it is convenient to analyze the role of the involved parameters into the different $S_{m}(T, B)$ trajectories. For such a purpose one can use the properties of the well known CMN salt available in the literature [3, 16, resumed in a $S_{m}(T, B)$ diagram in Fig. 2, In that figure it is evident that the entropy decrease, indicated as a doted curve at $T_{i}$, has a maximum slope at some intermediate value. This means that an optimum $\partial S_{m} /\left.\partial B\right|_{T_{i}}$ variation occurs at $k_{B} T=\mu_{B} B$ because thermal and Zeeman splitting become comparable. This condition can be traced in the 3D figure by following the maximum curvature of the $S_{m}(T, B)$ trajectory along different fields, indicated as a continuous curve in the figure.

Another characteristic is related with the size of the cooling window as a function of the applied field, as a

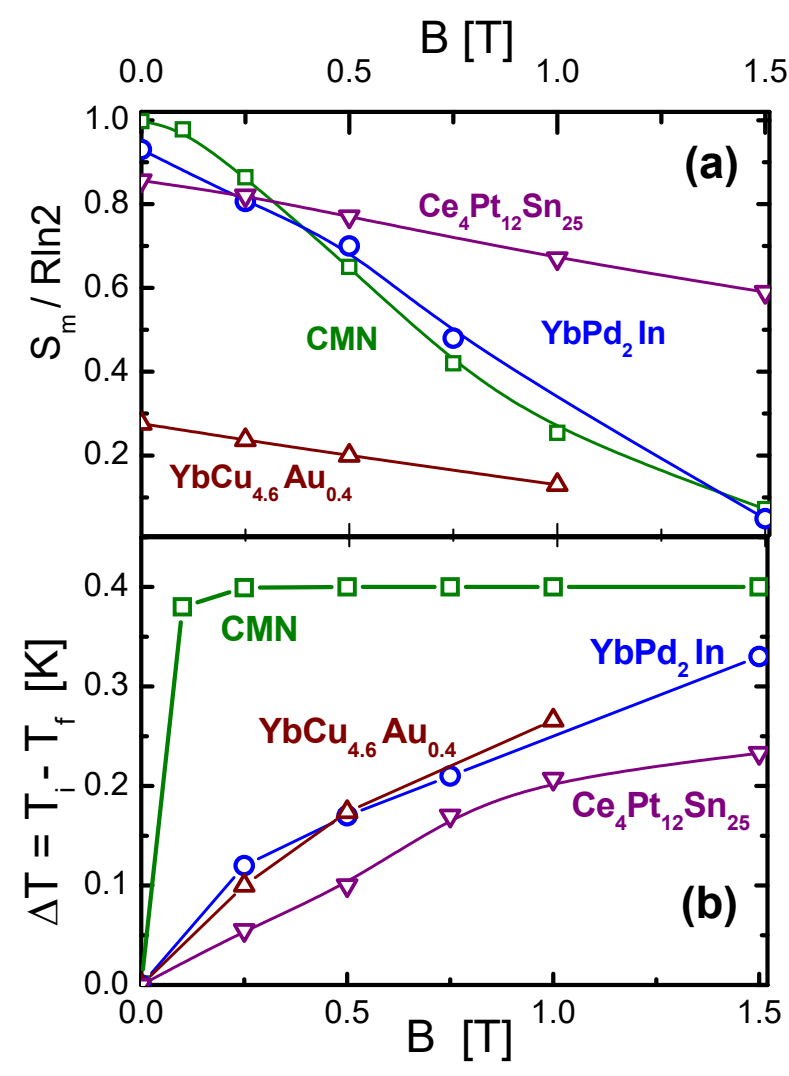

FIG. 3. Comparison of two characteristic parameters between $\mathrm{CMN}$ [3, $\mathrm{YbPd}_{2}$ in [6, $\mathrm{YbCu}_{4.6} \mathrm{Au}_{0.4}$ 8] and $\mathrm{Ce}_{4} \mathrm{Pt}_{12} \mathrm{Sn}_{25}$ [17. as a function of applied field: a) Field dependence of the entropy $S_{m}(B)$ at $0.4 \dot{\mathrm{K}}$ and b) cooling window $\Delta T$ at different fields in the adiabatic process.

sort of magneto-cooling efficiency: $\Delta T / \Delta B$. In the case of CMN one can see that $\Delta T$ already reaches $90 \%$ of its maximum value at around $B=0.25 \mathrm{~T}$ as it is indicated by a dashed line in Fig. 2. Therefore, further increase of field does not improve the cooling window. In this case, the initial temperature is taken as $T_{i}=0.4 \mathrm{~K}$ in order to present this feature in a convenient energy scale.

Both quantities are compared for different intermetallic compounds in Fig. 3 as a function of field. The different $S_{m}(B)$ trajectories presented in Fig. 3 a clearly indicate that $\mathrm{CMN}$ and $\mathrm{YbPd}_{2} \mathrm{In}$ are the most sensitive under applied magnetic field. Notably the low sensitivity of $\mathrm{YbCu}_{4.6} \mathrm{Au}_{0.4}$ and $\mathrm{Ce}_{4} \mathrm{Pt}_{12} \mathrm{Sn}_{25}$ is due to opposite reasons, the former because its ground state shows reminiscence of a liquid of spins [5] while the later because of its antiferromagnetic character 17 .

The field dependence of the cooling window $\Delta T$ is collected in Fig. 3b. As expected, CMN reaches its maximum value at very low field because its ordering temperature is $T_{N}=2 \mathrm{mK}$ whereas for intermetallic 


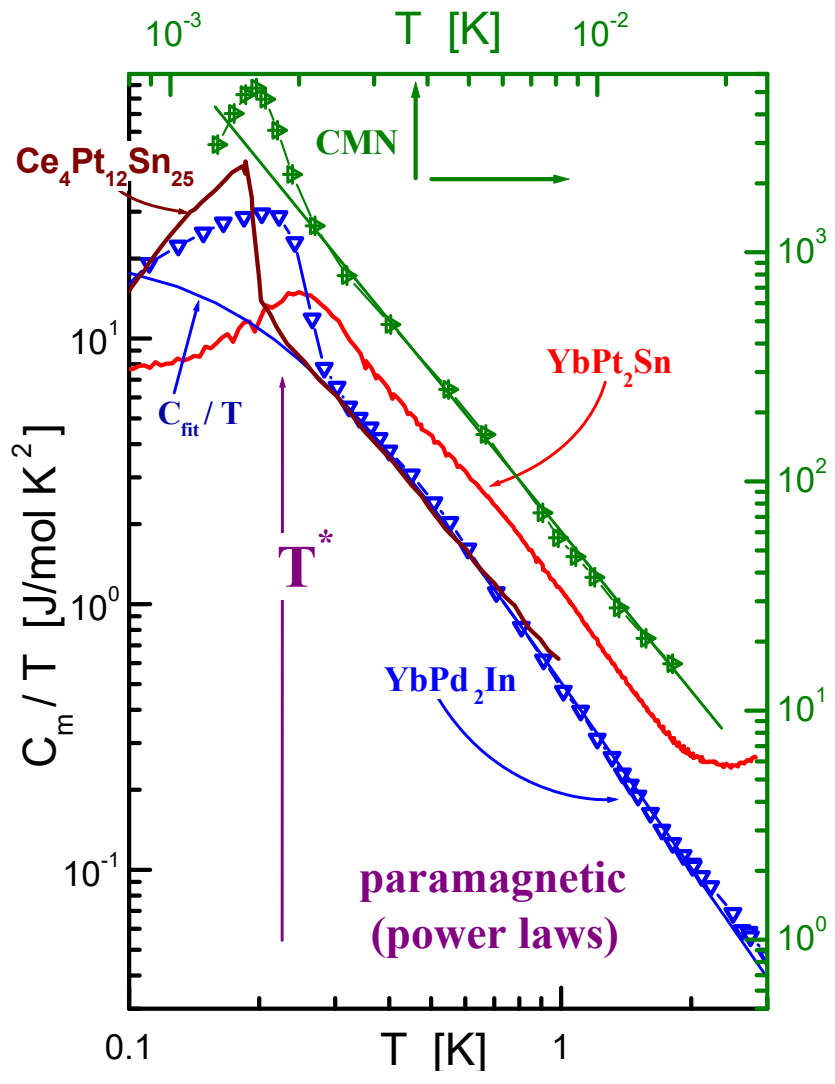

FIG. 4. Low temperature specific heat of some VHF in a double logarithmic scale. Left and lower axes: $\mathrm{YbPt}_{2} \mathrm{Sn}$ 9, $\mathrm{YbPd}_{2} \mathrm{In}$ [6] and $\mathrm{Ce}_{4} \mathrm{Pt}_{12} \mathrm{Sn}_{25}$ [17] with similar power law dependence (left and lower axes). Dashed curve represents the fit at $T>T^{*}$ with $C_{f i t} / T=0.5 /\left(T^{2.35}+0.02\right)$. Right and upper axes: CMN is included (after ref. [3]) for comparison and discussion, with the straight line representing a $T^{-2.3}$ dependence.

compounds $\Delta T$ increases gradually because their characteristic temperatures are quite comparable with the applied field.

From these comparisons, one concludes that the systems suitable for temperature stabilization (FTP) have lower capacity to control thermal drift but they are more sensitive to magnetic. The contrary occurs for those with higher capacity to absorb heat within a more extended range of temperature (CTD).

\section{THERMAL PROPERTIES OF VHF}

Turning our attention to the thermal properties of the VHF- $\mathrm{YbT}_{2} \mathrm{X}$ family of group (I), collected in Fig. 4 , one notices that they show the highest $C_{m} /\left.T\right|_{T \rightarrow 0}$ ratio among $\mathrm{Yb}$-intermetallics, reaching a maximum of

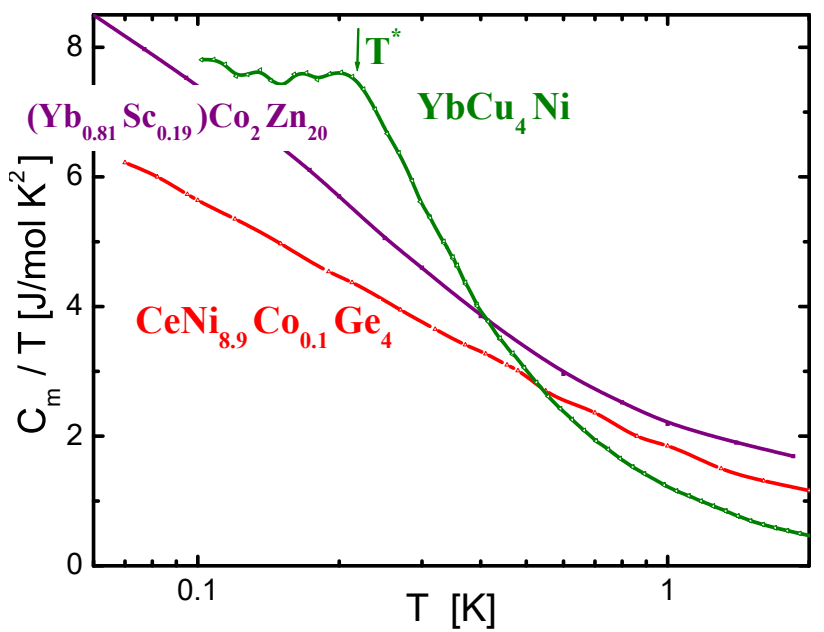

FIG. 5. Specific heat temperature dependence of some selected compounds from groups (II) and (III) in a semilogarithmic representation. $T^{*}$ marks the lower limit of the power law dependence for $\mathrm{YbCu}_{4} \mathrm{Ni}$.

$\approx 30 \mathrm{~J} / \mathrm{molK}^{2}$ at $T^{*} \approx 200 \mathrm{mK}$ after subtracting the Yb-nuclear contribution [5]. Among Ce-based intermetallic compounds, to our knowledge only one can be included into this group. These values are obviously exceeded by the Ce paramagnetic salt CMN. It is worth noting that these large $C_{m} /\left.T\right|_{T \rightarrow 0}$ values become quite similar if respective $C_{m}(T)$ dependencies are compared as it will be done in the following Section.

A common feature of their $C_{m}(T) / T$ increase at $T>$ $T^{*}$ is the power law thermal dependencies: $C_{f i t} / T=$ $G /\left(T^{D}+T_{0}^{D}\right)[18$ within the paramagnetic range, see for example the dashed curve in Fig. 4. They exhibit similar exponents: $2.27 \leq D \leq 2.35$ and $T_{0}$ values: $0 \leq T_{0} \leq 0.1$. In these compounds the slight deviation from a pure power law dependence is accounted by $T_{0}$ that can be attributed to a reminiscence of a very weak Kondo-type interaction. As expected, CMN shows a pure power law dependence because of the lack of conduction electrons.

For comparison with the members of other groups, the $C_{m}(T) / T$ of some representative compounds of group (II): $\mathrm{YbCu}_{4} \mathrm{Ni}$ [8, and group (III): $(\mathrm{Yb}, \mathrm{Sc}) \mathrm{Co}_{2} \mathrm{Zn}_{20}$ 7] and $\mathrm{CeNi}_{8.9} \mathrm{Co}_{0.1}$ [14 are collected in Fig. 5 in a logarithmic $T$ dependence. There one can see how the density of excitations is distributed in energy in NFL systems (with a $C_{m}(T) / T \propto$ $-\ln \left(T / T_{0}\right)$ dependence) respect to those with a power law $\left(C_{f i t}(T) / T \propto 1 / T^{1.24}[\right.$ [] $)$ like $\mathrm{YbCu}_{4} \mathrm{Ni}$ at $T>T^{*}$ also included into the figures. 


\section{MAGNETIC INTERACTIONS AND FRUSTRATION}

The absence of long range order is a 'sine qua non' condition for a cryo-material because it allows to keep large volume entropy (i.e. entropy per molar volume) down to very low temperature. Two main reasons may allow to retain the paramagnetic GS despite of the robust character of their magnetic moments: very weak magnetic exchange between neighboring spins mostly observed in group (I) 9] or magnetic frustration usually present in group (II) [8]. A third possibility occurs in the proximity to a quantum critical point that is related to the NFL behavior characterizing group (III) 7].

$\mathrm{YbT}_{2} \mathrm{X}$ compounds are the good representatives of weak magnetic exchange: $\aleph=J_{i, j} \times S_{i} S_{j}$ because the usual RKKY interaction in intermetallics depends on the conduction density of states: $\delta\left(\epsilon_{F}\right)$, becuase $J_{i, j}=J_{l o c} \delta\left(\epsilon_{F}\right) f\left(1 / d^{3}\right)$. The large residual resistivity of these Yb-based compounds, coincident with a small $\partial \rho / \partial T$ coefficient [9] and very low Sommerfeld coefficient $\gamma \approx \delta\left(\epsilon_{F}\right)$ observed in the non-magnetic reference compounds $\mathrm{LuPt}_{2} \mathrm{In} / \mathrm{Sn}$ converge to very low $\delta\left(\epsilon_{F}\right)$ values. In the CMN salt a RKKY interaction is certainly excluded because $\delta\left(\epsilon_{F}\right)=0$ and the expected dipolar interaction between quite distant Ce atoms decays exponentially.

The 3D geometrical frustration, favored by the pyrochlore structure of many components of group (II), may guarantee the lack of long range magnetic order but not the largest $C_{m} / T$ values (see e.g. [13]). Although the compounds of the NFL group (III) show more extended distribution of magnetic excitations, favoring the $R_{Q}(B)$ ratio, their proximity to a quantum critical point is related with a weakening of their magnetic moments with the consequent reduction of the $\partial M / \partial T=\partial S / \partial H$ slopes.

The maximum in $C_{m}(T) / T$, labelled as $T^{*}$ in Fig. 4 , may be regarded as a standard second order phase transition induced by long range magnetic interactions. However, in these systems more than $40 \%$ of the magnetic entropy is already condensed above $T^{*}$ and the divergent character of the $C_{m}\left(T>T^{*}\right) / T$ power law dependence points more likely to a so-called 'entropy bottleneck' effect 21] caused by the thermodynamic constraint in the entropy evolution imposed by the Nernst postulate: $\left.S_{m}\right|_{T \rightarrow 0} \geq 0$ [22]. This constraint induces relevant modifications in the entropy trajectory because it imposes a change of the free energy minimum. Depending of the nature of the alternative munimum (at lower temperature) the system may slide into different types of ordered phases that react differently under the application of magnetic field. $\mathrm{Ce}_{4} \mathrm{Pt}_{12} \mathrm{Sn}_{25}$ for example [17] shows a typical antiferromagnetic be-

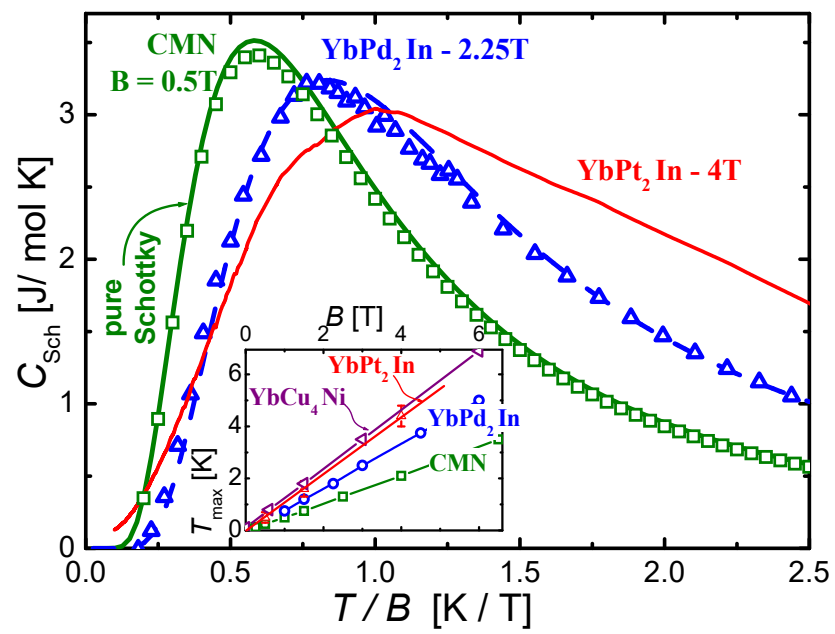

FIG. 6. a) Selected Schottky type anomalies of $C_{m}(T)$ scaled with respective applied fields. Inset: linear field dependence of the temperature of corresponding $C_{m}(T, B)$ maxima.

havior, whereas $\mathrm{YbPd}_{2} \mathrm{In}$ tends to a critical pint at $B \approx 0.75 \mathrm{~T}[6]$. Except for the Ce salt, where the magnetic interaction seems to be quite strong (and of dipole-dipole nature), the other systems with weak interactions show a similar behavior among them, transforming their specific heat jumps into the Schottky type anomalies once the external field has overcome the weak internal (molecular) field of the order of $\mu_{B} B \approx k_{B} T^{*}$. Some examples are collected in Fig. 6, where one may also note the similar values of their $C_{m}(T)$ that were mentioned in the previous section.

\section{MAGNETIC FIELD EFFECTS ON THE GROUND STATE}

The curves included in Fig. 6 are compared using a normalized $T / B$ abscissa selecting field that overcome the weak interactions governing the zero field ground state which cause the specific heat jumps observed in Fig. 4. These thermal characteristics imply that in the analyzed VHF compounds there is an equivalent field induced Zeeman splitting effect on the GS doublet. Furthermore, the observed scaling on ' $T / B^{\prime}$ at $B>0$ confirms the weak nature of the interactions present at $B=0$ because the $C_{m}(T)$ tendency to a Schottky type anomaly indicates that the ground state doublet behaves as that of a non interacting (or single) particle.

Notably, the $C_{m}(T)$ of CMN kindly fits into a pure Schottky anomaly for two 'Dirac' levels with the expected $C_{m}\left(T_{\max }\right)=3.6 \mathrm{~J} / \mathrm{molK}$ 24, whereas the two $\mathrm{YbT}_{2} \mathrm{In}$ intermetallics show a slightly broadened 
anomaly with a concomitant reduced $C_{m}\left(T_{\max }\right)$ value. This difference is likely due to the nature of the interctions dominating the GS at $B=0$. Taking profit that the temperature of respective maxima $T_{\max }(B)$ increases quite linearly with field, the giromagnetic factors of respective ground state doublets $g_{G S}$ can be evaluated from: $T_{\max }(B)=0.42 * g_{\text {eff }} * B *\left(\mu_{B} / k_{B}\right)$ [23]. These field dependencies are compared in the inset of Fig. 6 as a $T_{\max }(B)$ dependence. One should remark that no Schottky-like behavior is allowed down to $T \rightarrow 0$ because of Nernst postulate, which compels the system to reduce the two fold degeneracy of its GS through any alternative minimum within the free energy surface, like e.g. the transitions presented in Fig. 4 .

\section{SUMMARY}

From this analysis one concludes that different types of applications can be distinguished within the subKelvin range of temperature, recognized as those able to provide 'fix thermal points - FTP' (e.g. for photondetectors) and those for a 'controlled thermal drift CTD' (e.g. for standard cryostats). Since respective applications can be optimized by a proper choice of cryo-materials that show different types of entropy trajectories, a comparative analysis using simple characterizing parameters is proposed. One finds that, in the case of photon-detectors working around the $100 \mathrm{mK}$ range, they can be thermally stabilized using some recently discovered VHF compounds as FTP materials. The analysis of their cooling efficiency, $\Delta T$ vs. $\Delta B$, indicates that $90 \%$ of the thermal window is already reached at relatively low fields $(B \leq 1 \mathrm{~T})$. This family of Yb-based compounds from group (I) show high volume entropy and convenient sensitivity to magnetic field variation because of a large $\partial M / \partial T$. The field dependence of their paramagnetic GS can be simply described using a two level Schottky anomaly for the specific heat once the applied field has overcome their weak GS interactions. Complementary, the compounds of groups (II) and (III), with softer $C_{m}(T) / T$ dependencies show higher capacity for heat absorption $Q_{a b s}$ distributed in a larger range of temperature as required for CTD applications. Although they are not so sensitive to magnetic field variation, one may conclude that there is not a unique 'best' system for ADR but different optimal choices depending on the type and range of application. In this case one may remark that paramagnetic salts do not offer suitable compounds for CTD as provided by intermetallic ones.

From the analysis of the physical phenomena occurring in the range of energy where quantum effects become dominant one learns that, while quantum mechanisms intervenes in the formation of alternative GS, the Nernst postulate imposes conditions on their thermodynamic feasibility as $\rightarrow 0$. This means that quantum mechanics and thermodynamic laws work entangled providing and defining the proper ground state.
[1] B. Collaudin and N. Rando, in Cryogenics in space: a review of the missions and of the technologies, Cryogenics 40 (2000) 797.

[2] R.T. Kouzes and J.H. Ely, in Status summary of $\mathrm{He}^{3}$ and neutron detection alternatives for homeland security Report PNNL- 19360, Pacific Northwest National Laboratory, 2010.

[3] W.F. Giauque, R.A. Fisher, E.W. Hornung, G.E. Brodale, in Magnetothennodynamics of $\mathrm{Ce}_{2} \mathrm{Mg}_{3}\left(\mathrm{NO}_{3}\right)_{12} .24 \mathrm{H}_{2} \mathrm{O}$, J. Chem. Phys. 58 (1973) 2621.

[4] F. Pobell, in Matter and Methods at Low Temperatures, Springer-Verlag (1991).

[5] I. Curlìk, M. Giovannini, J.G. Sereni, S. Gabani, M. Reiffers, in Extremely high density of magnetic excita-

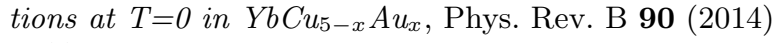
224409 .

[6] F. Gastaldo, A. Dzubinskà, M. Reiffers, G. Pristàs, I. Curlìk, J.G. Sereni, M. Giovannini, in $\mathrm{YbPd}_{2}$ In: $a$ promising candidate to strong entropy accumulation at very low temperature, ArXiv [cond-mat] 1711.02335, 27 June 2018.

[7] Y. Tokiwa, B. Piening, H.S. Jeevan, S.L. Budko, P.C. Canfield, P. Gegenwart,in Super-heavy electron mate- rial as metallic refrigerant for adiabatic demagnetization cooling, Sci. Adv. 2 (2016) e1600835.

[8] J.G. Sereni, I. Curlk, M. Giovannini, A. Strydom, M. Reiffers, in Physical properties of the very heavy fermion $\mathrm{YbCu}$ A $\mathrm{Ni}$ arXiv:1805.08051v1 [cond-mat.str-el] 21 May 2018

[9] T. Gruner, D. Jang, A. Steppke, M. Brando, F. Ritter, C. Krellner, C. Geibel, in Unusual weak magnetic exchange in $\mathrm{YbPt}_{2} \mathrm{Sn}$ and $\mathrm{YbPt}_{2} \mathrm{In}$, J. Phys.: Condens. Matter 26485002 (2014).

[10] D. Jang, T. Gruner, A. Steppke, K. Mistsumoto, C. Geibel, M. Brando, in Large magnetocaloric effect and adiabatic demagnetization refrigeration with $\mathrm{YbPt}_{2} \mathrm{Sn}$, Nature Communications, ncomms9680 (2015).

[11] J.G. Sereni, in Entropy constraints in the ground state formation of magnetically frustrated systems, J. Low Temp. Phys. 190 (2018) 1-19, and references therein.

[12] G.R. Stewart, in Non-Fermi-liquid behavior in $d$ - and f-electron metals, Rew. Mod. Phys. 73 (2001) 797.

[13] J.G. Sereni, in Role of the entropy in the ground state formation of magnetically frustrated systems, Physica B: Condensed Matter 536 (2018) 397.

[14] L. Peyker, C. Gold, W. Scherer, H. Micho, EW. Scheidt, in Competing magnetic interactions in 
$\mathrm{CeNi}_{9-x} \mathrm{Co}_{x} \mathrm{Ge}_{4}$, J. of Phys.: Conf. Series 273 (2011) 012049.

[15] L. Peyker, C. Gold, E-W. Scheidt, W. Scherer, J.G. Donath, P. Gegenwart, F. Mayr, T. Unruh, V. Eyert, E. Bauer, H Michor, in Evolution of quantum criticality in $\mathrm{CeNi}_{9-x} \mathrm{Cu}_{x} \mathrm{Ge}_{4}$, J. of Phys.: Cond. Mat. 21 (2009) 235604.

[16] R. A. Fisher, E. W. Hornung, G. E. Brodale, and W. F. Giauque, in Magnetothermodynamics of $\mathrm{Ce}_{2} \mathrm{Mg}_{3}\left(\mathrm{NO}_{3}\right)_{12} .24 \mathrm{H}_{2} \mathrm{O}$, J. Chem. Phys. 58 (1973) 5584 .

[17] N. Kurita, H. Lee, Y. Tokiwa, C. F. Miclea, E. D. Bauer, F. Ronning, J. D. Thompson, Z. Fisk, P. Ho, M. B. Maple, P. Sengupta, I. Vekhter, R. Movshovich, in Thermal and magnetic properties of the low-temperature antiferromagnet $\mathrm{Ce}_{4} \mathrm{Pt}_{12} \mathrm{Sn}_{25}$, Phys. Rev. B 82 (2010) 174426.

[18] J.G. Sereni, in Peculiar thermal features of Ce-systems around their critical points J. Low Temp. Phys. 147 (2007) 179.

[19] R. Moessner and A.P. Ramirez, in Geometrical Frustration; Physics Today, February 2006, p.24.
[20] M.S. Torikachvili, S. Jia, E.D. Mun, S.T. Hannahs, R.C. Black, W.K. Neils, D. Martien, S.L. Bud'ko, P.C. Canfield, in Six closely related related $Y_{b} T_{2} Z n_{20}$ heavy fermion compounds with large local moment degeneracy, PNAS 104 (2007) 9960.

[21] J.G. Sereni, in Entropy Bottlenecks at $T \rightarrow 0$ in CeLattice and Related Compounds, J Low Temp Phys 179 (2015) 126.

[22] A.B. Pippard, in Elements of classical Thermodynamics, University Press, Cambridge, 1964.

[23] T. Klimczuk, C.H. Wang, J.M. Lawrence, Q. Xu, T. Durakiewicz, F. Ronning, A. Llobet, F. Trouw, N. Kurita, Y. Tokiwa, Han-oh Lee, C.H. Booth, J.S. Gardner, E. D. Bauer, J.J. Joyce, H.W. Zandbergen, R. Movshovich, R.J. Cava, J.D. Thompson, in Crystal fields, disorder, and antiferromagnetic short-range order in $\mathrm{Yb}_{0.24} \mathrm{Sn}_{0.76} \mathrm{Ru}$, Phys. Rev. B 84 (2011) 075152.

[24] J.G. Sereni, in: Magnetic Systems: Specific Heat, Saleem Hashmi (editor-in-chief), Materials Science and Materials Engineering. Oxford: Elsevier; 2016. pp. 113; ISBN: 978-0-12-803581-8. 